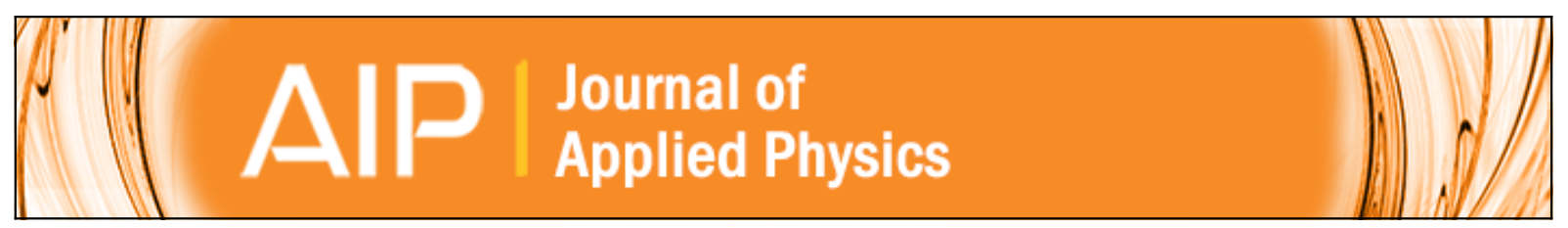

\title{
Third-order nonlinear optical response of colloidal gold nanoparticles prepared by
} sputtering deposition

Hemerson P. S. Castro, Heberton Wender, Márcio A. R. C. Alencar, Sergio R. Teixeira, Jairton Dupont, and Jandir M. Hickmann

Citation: Journal of Applied Physics 114, 183104 (2013); doi: 10.1063/1.4831679

View online: http://dx.doi.org/10.1063/1.4831679

View Table of Contents: http://scitation.aip.org/content/aip/journal/jap/114/18?ver=pdfcov

Published by the AIP Publishing

\section{Articles you may be interested in}

Huge enhancement of optical nonlinearities in coupled $\mathrm{Au}$ and $\mathrm{Ag}$ nanoparticles induced by conjugated polymers Appl. Phys. Lett. 100, 023106 (2012); 10.1063/1.3675911

Formation of ultrafine uniform gold nanoparticles by sputtering and redeposition

Appl. Phys. Lett. 94, 133107 (2009); 10.1063/1.3111443

Nonlinear optical properties of Au nanoparticles colloidal system: Local and nonlocal responses

Appl. Phys. Lett. 92, 201902 (2008); 10.1063/1.2929385

Combinatorial investigation of the isolated nanoparticle to coalescent layer transition in a gradient sputtered gold nanoparticle layer on top of polystyrene

Appl. Phys. Lett. 88, 021910 (2006); 10.1063/1.2161926

Publisher's Note: "Enhancement of third-order nonlinear optical susceptibilities in silica-capped Au nanoparticle films with very high concentrations" [Appl. Phys. Lett.84, 4938 (2004)]

Appl. Phys. Lett. 85, 509 (2004); 10.1063/1.1776193

High-Voltage Amplifiers

- Voltage Range from $\pm 50 \mathrm{~V}$ to $\pm 60 \mathrm{kV}$

- Current to 25A

Electrostatic Voltmeters

- Contacting \& Non-contacting

- Sensitive to $1 \mathrm{mV}$

- Measure to $20 \mathrm{kV}$
ENABLING RESEARCH AND

INNOVATION IN DIELECTRICS,

ELECTROSTATICS,

MATERIALS, PLASMAS AND PIEZOS

TRek www.trekinc.com 


\title{
Third-order nonlinear optical response of colloidal gold nanoparticles prepared by sputtering deposition
}

\author{
Hemerson P. S. Castro, ${ }^{1}$ Heberton Wender, ${ }^{2,3}$ Márcio A. R. C. Alencar, ${ }^{1, a)}$ \\ Sergio R. Teixeira, ${ }^{4}$ Jairton Dupont ${ }^{5}$ and Jandir M. Hickmann ${ }^{1}$ \\ ${ }^{1}$ Optics and Materials Group-OPTMA, Universidade Federal de Alagoas, CAIXA POSTAL 2051, \\ 57061-970 Maceió, Brazil \\ ${ }^{2}$ Brazilian Synchrotron National Laboratory (LNLS), CNPEM, Rua Giuseppe Máximo Scolfaro 10.000, \\ 13083-970 Campinas, Brazil \\ ${ }^{3}$ Department of Physics, Universidade Federal do Mato Grosso do Sul, 79070-900, Campo Grande, Brazil \\ ${ }^{4}$ Institute of Physics, Universidade Federal do Rio Grande do Sul, 91501-970, Porto Alegre, Brazil \\ ${ }^{5}$ Laboratory of Molecular Catalysis, Institute of Chemistry, Universidade Federal do Rio Grande do Sul, \\ 91501-970, Porto Alegre, Brazil
}

(Received 15 July 2013; accepted 1 November 2013; published online 13 November 2013)

\begin{abstract}
The nonlinear optical responses of gold nanoparticles dispersed in castor oil produced by sputtering deposition were investigated, using the thermally managed Z-scan technique. Particles with spherical shape and $2.6 \mathrm{~nm}$ of average diameter were obtained and characterized by transmission electron microscopy and small angle X-ray scattering. This colloid was highly stable, without the presence of chemical impurities, neither stabilizers. It was observed that this system presents a large refractive third-order nonlinear response and a negligible nonlinear absorption. Moreover, the evaluation of the all-optical switching figures of merit demonstrated that the colloidal nanoparticles prepared by sputtering deposition have a good potential for the development of ultrafast photonic devices. (C) 2013 AIP Publishing LLC. [http://dx.doi.org/10.1063/1.4831679]
\end{abstract}

\section{INTRODUCTION}

Colloidal metallic nanoparticles have been intensively investigated in recent years. The reason for this great interest is related to the astounding physical responses that these systems provide when they interact with light sources, due to the excitation of localized surface plasmon resonances (LSPRs). ${ }^{1,2}$ In many cases, the LSPR generates a huge enhancement of the local field in the surroundings of the nanoparticles, which raises the nonlinear optical responses of the colloidal medium. ${ }^{2-15}$ Owing to this fact, different proposals have been made in order to exploit this effect for the development of different optical and photonic applications, such as surface enhanced Raman spectroscopy (SERS), ${ }^{4}$ optical limiters, ${ }^{4-6}$ and optical switching devices. ${ }^{5,6}$

In particular, aiming ultrafast all-optical switching applications, it is necessary to develop a suitable colloid, combining the liquid host and metallic particles' absorptive and refractive responses. Usually, the composite's effective nonlinear refractive index, $n_{2 e f f}$, can be modified by changing the nanoparticles filling factor $f$. However, changing the value of $f$ also affects the materials' linear and nonlinear absorptions. Due to this fact, this ideal composite material has to be designed in order to present large enough $n_{2 \text { eff }}$ to perform optical switching operations in thicknesses comparable to the absorption length. Moreover, the unavoidable increase on linear and nonlinear absorptions induced by the metallic particles should not compromise the device's performance. This can be evaluated quantitatively by the calculation of the figures of merit (FOM), $W=\Delta n_{\max } / \lambda \alpha_{0}$ and

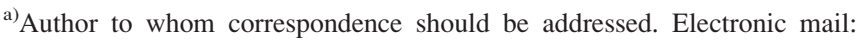
marca@optma.org
}

$T=2 \alpha_{2} \lambda / n_{2}$, where $\Delta n_{\max }$ is the maximum refractive index change achievable, limited by saturation. A good material for all-optical switching must satisfy simultaneously the conditions $|W|>0.27$ and $|T|<1 .^{16}$

On the other hand, it is known that the dielectric function of composite media depends strongly on the dielectric functions of the host and the inclusion, as well as on the particles' shape, size, and filling factor. For spherical particles, in particular, the composite linear absorption coefficient $\left(\alpha_{0}\right)$ is related to the real and imaginary parts of the inclusion first-order optical susceptibility, varying linearly with the particles filling factor $(f)^{17}$

$$
\alpha_{0}(\lambda)=\frac{18 \pi f}{\lambda} \varepsilon_{h}(\lambda)^{3 / 2} \frac{\varepsilon_{m}^{\prime \prime}(\lambda)}{\left[\varepsilon_{m}^{\prime}(\lambda)+2 \varepsilon_{h}(\lambda)\right]^{2}+\varepsilon_{m}^{\prime}(\lambda)},
$$

where $\lambda$ is the light wavelength, $\varepsilon_{h}$ is the host dielectric function, $\varepsilon_{m}^{\prime}$ and $\varepsilon_{m}^{\prime \prime}$ are the real and the imaginary parts of the particles dielectric function, respectively.

Similar behavior is also observed for the nonlinear refractive index and absorption coefficient. Previous works have demonstrated that, in composite medium, the presence of nanometric inclusions can modify strongly the material's nonlinear response. ${ }^{3,18}$ In this case, both host and inclusion linear and nonlinear optical properties contributes to the overall behavior. Indeed, the larger the amount of the inclusion the more significant is the modification on the composite response in comparison with the neat host. In addition, if the inclusions are consisted of metallic nanoparticles, the excitation of the localized surface plasmon resonance add an extra contribution to the composite response, due to the enhancement of the local field near the particles surface. 
For systems containing spherical particles at very low concentration, $f \ll 1$, the third-order nonlinear susceptibilities of the host, $\chi_{H}^{(3)}$, and individual particles, $\chi_{N P}^{(3)}$, are related to the composite effective nonlinear susceptibility by $^{17}$

$$
\chi_{e f f}^{(3)} \cong f \eta|\eta|^{2} \chi_{N P}^{(3)}+\chi_{H}^{(3)},
$$

where $\eta=3 \varepsilon_{H}(\lambda) /\left[\varepsilon_{N P}(\lambda)+2 \varepsilon_{H}(\lambda)\right], \varepsilon_{H}(\lambda)$ and $\varepsilon_{N P}(\lambda)$ are the host and the nanoparticle dielectric functions, respectively. As the nonlinear refractive index and absorption coefficient are related to the medium real and imaginary parts of the third-order susceptibility by

$$
n_{2}=\frac{3 \operatorname{Re}\left[\chi^{(3)}\right]}{4 \varepsilon_{0} n_{0}^{2} c}
$$

and

$$
\alpha_{2}=\frac{3 \omega \operatorname{Im}\left[\chi^{(3)}\right]}{2 \varepsilon_{0} n_{0}^{2} c}
$$

It can be shown that for this kind of composite material

$$
n_{2 e f f}=\frac{3\left\{f \operatorname{Re}\left[\eta|\eta|^{2} \chi_{N P}^{(3)}\right]+\operatorname{Re}\left[\chi_{H}^{(3)}\right]\right\}}{4 \varepsilon_{0} n_{0}^{2} c}
$$

and

$$
\alpha_{2}=\frac{3 \omega\left\{f \operatorname{Im}\left[\eta|\eta|^{2} \chi_{N P}^{(3)}\right]+\operatorname{Im}\left[\chi_{H}^{(3)}\right]\right\}}{2 \varepsilon_{0} n_{0}^{2} c}
$$

Combining Eqs. (2), (5), and (6), it can be observed that the FOM, $W$, and $T$ are strongly affected by the real and imaginary parts of the inclusion and host's linear and nonlinear susceptibilities. Therefore, for an accurate evaluation of the medium potential aiming all-optical switching application, the knowledge of the nanoparticles susceptibility is essential.

Using Eqs. (5) and (6), several authors obtained the third-order nonlinear susceptibilities of nanoparticles produced by different methods. ${ }^{14,19-26}$ Nevertheless, the use of this procedure to characterize particles produced by wet chemistry methods can lead to inaccurate results. In fact, the nanoparticles' production based on chemical reactions intrinsically results in colloidal systems containing byproducts, stabilizing agents, and remaining substrates. ${ }^{27}$ This happens due to the nature of the employed reduction of the corresponding metal salt or complexes, which is conducted in the presence of additional stabilizing agents to avoid substantial growth. Indeed, the presence of chemical impurities near the particles surface basically modifies the surrounding environment experienced by the latter. As the localized surface plasmon resonance phenomenon depends strongly with the particles neighborhood, its frequency as well as the field enhancement factor will be affected by the impurities. Therefore, the optical responses, linear and nonlinear, of a colloidal system are influenced by the presence of chemical impurities, even at small amounts. Hence, as the colloidal nanoparticles linear and nonlinear optical properties depend strongly with the surrounding medium, ${ }^{17}$ the reported values of the nonlinear susceptibility are probably contaminated by the additional compounds present in these colloids. Moreover, if those impurities have lower damage threshold in comparison with the host and particles, their presence may limit the use of the colloidal system for nonlinear optical applications as well.

In this work, we investigated the nonlinear optical response of colloids produced by sputtering deposition of $\mathrm{Au}$ nanoparticles (AuNPs) directly onto castor oil without using any stabilizing agent. ${ }^{27-34}$ The electronic contribution to the nonlinear refraction of these colloids were evaluated using the Z-scan technique with thermal management and the alloptical switching figures of merit for this composite system were also obtained. In addition, the third-order nonlinear susceptibility of AuNPs in an organic dispersant without the presence of other chemical impurities was obtained. Our results show that pure colloidal metallic AuNPs produced by this physical preparation method are promising materials for ultrafast nonlinear optical applications.

\section{EXPERIMENTAL}

The AuNPs were prepared following the same sputtering procedure reported before. ${ }^{28}$ Castor oil was chosen as liquid substrate for sputtering deposition in order to produce stable colloids without using other chemical reagents rather than the oil and the Au target. Moreover, this vegetable oil presents negligible third-order nonlinear optical responses at near infrared region. ${ }^{14,35}$ Hence, the colloid nonlinear refractive index and absorption coefficient depend mainly on the third-order nonlinear susceptibility of the metallic particles. The oil was degassed for approximately $3 \mathrm{~h}$ at $298 \mathrm{~K}$ under vacuum prior to being introduced into the sputtering machine (MED 020, Bal-Tech). Deposition was performed for $5 \mathrm{~min}$ in the following conditions: $300 \mathrm{~V}$ and $20 \mathrm{~mA}$ of applied voltage and current with an argon pressure of $2 \mathrm{~Pa}$ at room temperature. In each deposition, a mass of $1.2 \mathrm{~g}$ of castor oil was placed on a Petri plate ( $3 \mathrm{~cm}$ of diameter) and horizontally set in the sputter coater. The liquid surface was located at a distance of $50 \mathrm{~mm}$ from the gold target $(99.99 \%$ in purity).

The AuNPs size and shape were characterized by transmission electron microscopy (TEM) and small angle X-ray scattering (SAXS) techniques. For TEM measurements, the samples were prepared by dispersion of the AuNPs in isopropanol at room temperature and then collected on a carboncoated copper grid.

SAXS experiments were performed in the colloids at the SAXS1 beam line of Brazilian Synchrotron Light Laboratory (LNLS) with $\lambda=1.488 \AA$ and $0.05<Q<3.33 \mathrm{~nm}^{-1}$, where $Q=4 \pi \sin (2 \theta) / \lambda ; Q$ is the scattering vector, $\theta$ is the scattering angle, and $\lambda$ is the $\mathrm{X}$-ray wavelength. The colloidal solutions were injected by a syringe into a cell with mica windows specially designed for SAXS experiments in liquids. The fitting procedure of the experimental SAXS data was made 
using the SASfit program by minimizing the squared chi $\left(\chi^{2}\right)^{31}$

For nonlinear optical characterization, a mode-locked Ti-Sapphire laser, delivering pulses of $200 \mathrm{fs}$ at a $76 \mathrm{MHz}$ repetition rate, tuned at $793 \mathrm{~nm}$ was employed as the excitation source. The laser beam was modulated by a chopper $(14 \mathrm{~Hz})$ and focused onto the sample by a convergent lens $7.5 \mathrm{~cm}$ focal length. The samples consisted of $1 \mathrm{~mm}$ width quartz cell filled with the investigated colloid. The cell was mounted on a translation stage and moved around the lens focal plane $(\mathrm{z}=0)$. The light transmittance was then measured by a closed-aperture photodetector as a function of the sample position. The detected signal was temporally analyzed by digital oscilloscope and then processed by a computer.

Nonlinear absorption measurements were performed with the same experimental setup but using a configuration without aperture and chopper. In this case, the laser repetition rate was reduced to $1 \mathrm{kHz}$ by a Pockels cell and the measured transmittance signal was processed by a lock in amplifier.

Owing to the laser large repetition rate, the cumulative thermo-optical effect dominates the refractive response of the medium after some time of the sample being irradiated. In this experimental condition, we must use a variation of $\mathrm{Z}$-scan technique that manages the thermal induced nonlinear effects. ${ }^{36,37}$ Hence, it is possible to separate the thermal and the electronic contributions to the observed nonlinear refraction, analyzing the transmittance signal at different time scales.

First, we define $t=0$ as the time instant that the chopper begins unblock the laser beam. At time instants much larger than the chopper rise time, the thermal response dominates and the measured transmittance in Z-scan technique can be expressed by ${ }^{37}$

$$
T(z, t)=1+\theta \operatorname{Tan}^{-1}\left[\frac{2 q\left(z / z_{0}\right)}{\left[(2 q+1)^{2}+\left(z / z_{0}\right)^{2}\right] \frac{t_{c}(z)}{2 q t}+2 q+1+\left(z / z_{0}\right)^{2}}\right],
$$

where $\theta$ is the thermal induced phase-shift, $z_{0}$ corresponds to the Rayleigh range of the laser beam, $q$ is the order of the multiphoton process, $t_{c}(z)=w_{0}^{2}\left(1+\left(z / z_{0}\right)^{2}\right) / 4 D$ is the characteristic thermal lens time, $w_{0}$ is the beam waist at the focal plane, and $D$ is the medium thermal diffusivity.

During the time period between $t=0$ and the chopper rise time, the laser beam is partially blocked in such a way that the beam power on the sample varies with time. Therefore transmittance measurements within this period cannot be described by the Eq. (7) and are disregard at the analysis procedure. However, the temporal evolution of the Z-scan traces can be followed from the opening rise time onwards. ${ }^{36}$

Ideally, the electronic contribution to the observed nonlinear refraction, $n_{2}$, gives an instantaneous response. Although we could not measure the normalized transmittance at $t=0$, we can reconstruct this curve extrapolating the time evolution curves of the measured normalized transmittance, at all sample positions, using Eq. (7). ${ }^{37}$ Hence, the value of $n_{2}$ can be obtained fitting the normalized transmittance curve at $t=0$ employing the standard equation of Zscan method ${ }^{38}$

$$
T(z) \cong 1+\frac{4 \Delta \Phi_{0}\left(z / z_{0}\right)}{\left[\left(z / z_{0}\right)^{2}+9\right]\left[\left(z / z_{0}\right)^{2}+1\right]},
$$

where $\sqrt{2} \Delta \Phi_{0}=k n_{2} I_{0} L_{\text {eff }}$ is the nonlinear phase shift, $L_{\text {eff }}=\left(1-e^{-\alpha_{0} L}\right) / \alpha_{0}$ is the sample effective length, $\alpha_{0}$ is the linear absorption coefficient of the colloid, and $L$ is the sample length.

For nonlinear absorption measurements, the transmittance curve was normalized dividing the measured values by the transmitted intensity when the sample was positioned far away from the lens focal plane. Thus, the nonlinear absorption coefficient can be obtained by fitting the results using the equation ${ }^{38}$

$$
T(z)=\sum_{m=0}^{\infty} \frac{\left[-q_{0}(z, 0)\right]^{m}}{(m+1)^{3 / 2}},
$$

where $z$ is the sample position with respect to the lens focal plane, $q_{0}(z, t)=\beta I_{0}(t) L_{e f f} /\left(1+z^{2} / z_{0}^{2}\right), \beta$ is the material nonlinear absorption coefficient, $I_{0}(t)$ is the laser intensity at $z=0, z_{0}=\pi w_{0}^{2} / \lambda$ is the beam Rayleigh length, $w_{0}$ is the minimum beam waist, and $\lambda$ is the laser wavelength.

\section{RESULTS AND DISCUSSION}

In Figures 1(a) and 1(b), it is presented a typical TEM image of the produced particles and the corresponding particle's diameter histogram. The histograms of the AuNPs were obtained from the measurement of more than 300 particles and were reproduced in different regions of the $\mathrm{Cu}$ grid, found in arbitrarily chosen areas of enlarged micrographs. As can be observed by TEM, the particles presented spherical shapes with an average diameter of $3.6 \pm 0.7 \mathrm{~nm}$.

Figure 1(c) shows the intensity as function of the scattering vector $Q$ and the respective fit of the experimental data. By analyzing the scattering curve, it can be observed that the AuNPs do not form agglomerates, since the scattering intensity do not increase for low $Q$, and are non-interacting due to the absence of an structure factor. The size distribution could be obtained by fitting the scattering intensity by considering a form factor of spheres with a Lognormal size distribution, 

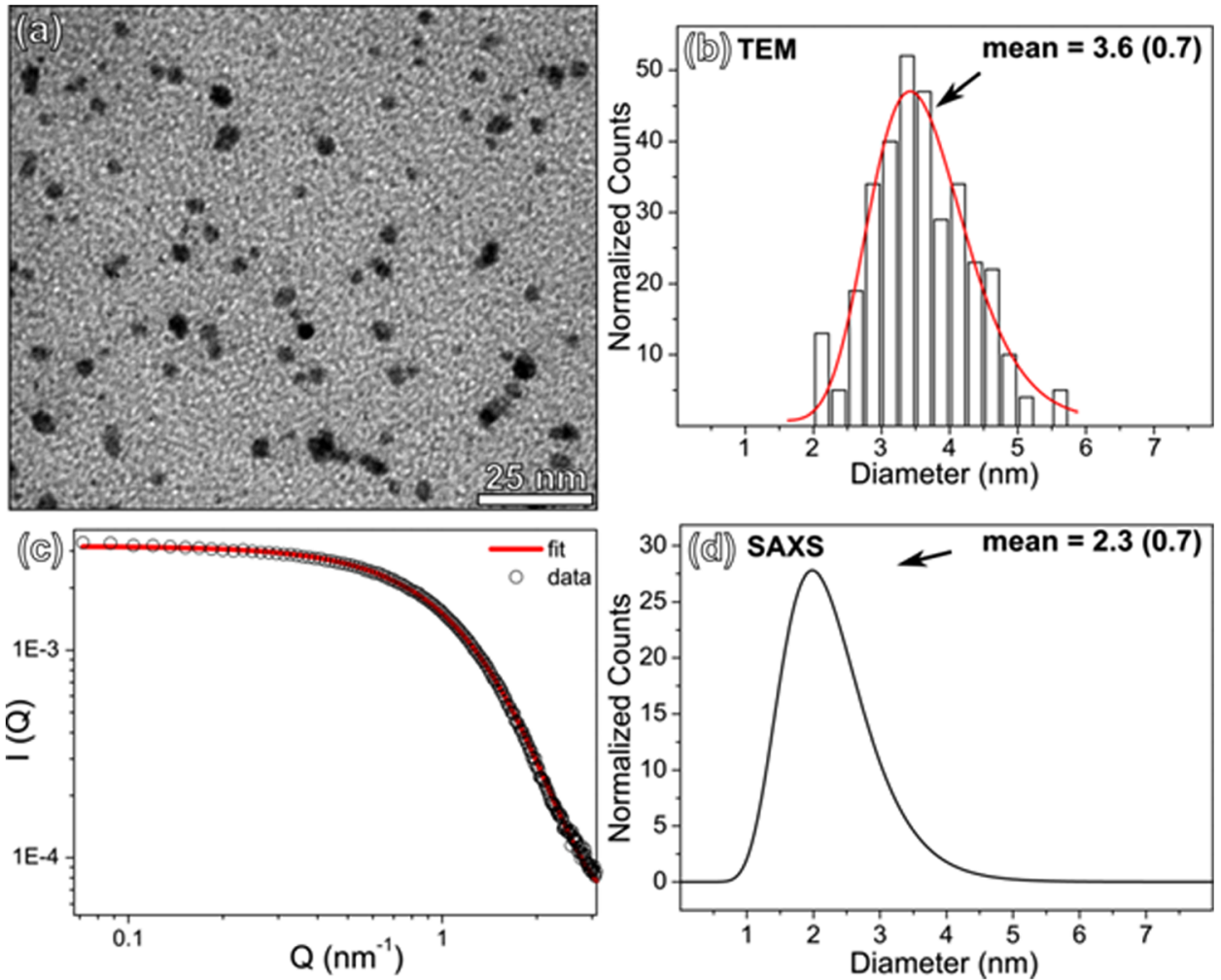

FIG. 1. (a) TEM image and (b) respective histogram showing the particle size distribution of AuNPs in castor oil. (c) SAXS curve and fitting of AuNPs in castor oil and (d) the corresponding size distribution obtained by considering spheres with a Lognormal size distribution in the form factor.
Figure 1(d). The result showed a mean diameter of $2.3 \pm 0.7 \mathrm{~nm}$, which is slight lower that the value obtained by TEM $(3.6 \pm 0.7 \mathrm{~nm})$. This difference may be due to the high accuracy of SAXS for measuring the particle's size since the scattering intensity carries contributions of all present particles and not only a limited number as in the case of TEM. Also there is no difficult in counting the smaller particles by SAXS, what is not the case of TEM. In fact, it is not discarded the possibility of particles coarsening after dilution of the AuNPs in castor oil with isopropanol during TEM analysis, since the relative amount of isopropanol was high enough for such effect in nanoparticles size. ${ }^{39}$

From the produced Au colloids, three different concentrations were obtained by diluting the mother solution adding known amounts of Castor Oil. Using this approach, three

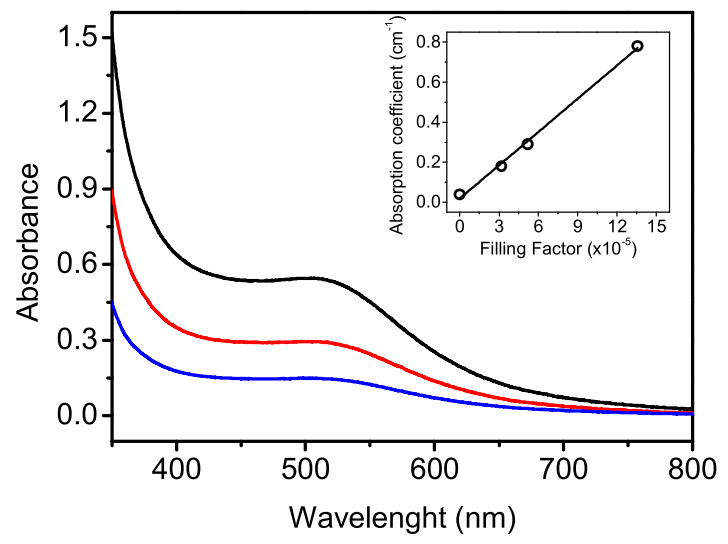

FIG. 2. UV-VIS-NIR absorption spectra of samples A (red line), B (green line), and $\mathrm{C}$ (blue line). The inset shows the variation of the castor oil and colloids linear absorption coefficient with the particles filling factor. colloids were prepared, namely, samples A, B, and C, with $1.36 \times 10^{-4}, 5.18 \times 10^{-5}$, and $3.19 \times 10^{-5}$ filling factors, respectively. In Figure 2, it is presented the absorption spectra of these systems. A resonance band, centered at $504 \mathrm{~nm}$, associated to the nanoparticles' localized surface plasmon is observed. In this figure inset, it is plotted the castor oil and the colloid absorption coefficients at $793 \mathrm{~nm}$, as a function of the particles' filling factor. A clear linear relation between these quantities is obtained, which indicates that the produced particles present spherical shape, which corroborates to the TEM and SAXS analysis.

In Figure 3, it is shown the results for the peak-valley transmittance variation as a function of the time, for all samples, which were obtained from the thermally managed Z-scan measurements. It can be observed that the presence of metallic nanoparticles increases significantly the modulus

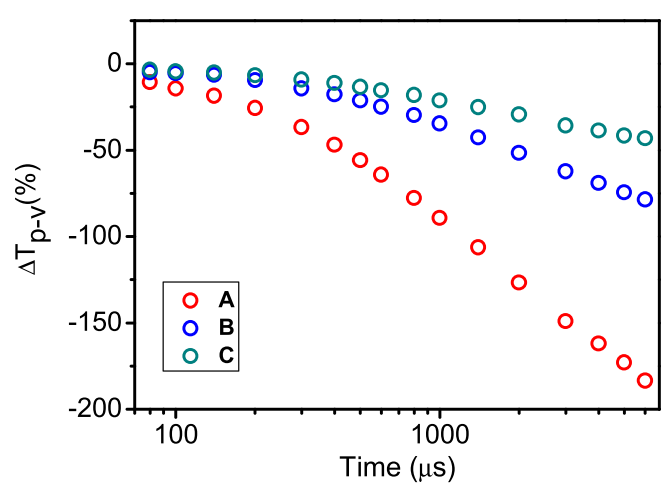

FIG. 3. Peak-valley transmittance variation as a function of the time for samples A (red open circles), B (green open circles), and C (blue open circles). The employed average laser powers were $15 \mathrm{~mW}, 11 \mathrm{~mW}$, and 10 $\mathrm{mW}$, respectively. 

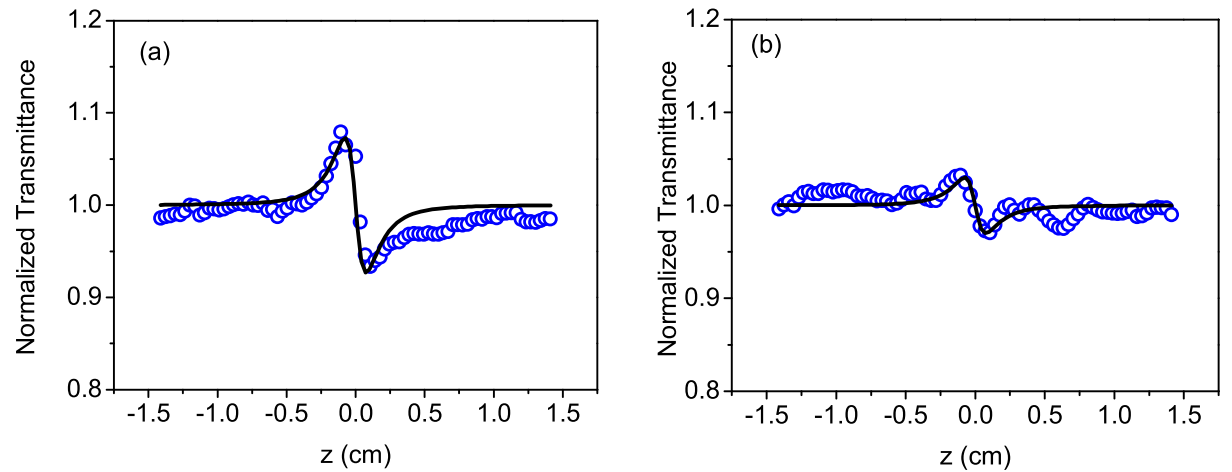

FIG. 4. Reconstructed Z-scan curves obtained at $t=0$ for the samples (a) A, (b) B, and (c) C, and (d) the openaperture measurement result for the
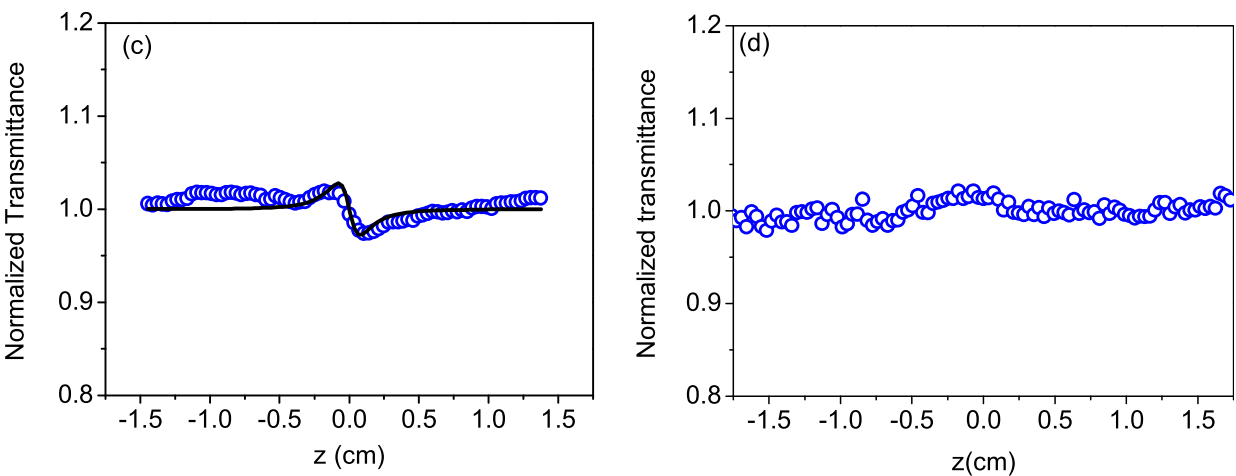
mother solution. The light intensities at $\mathrm{z}=0$ plane were $2.8 \times 10^{8} \mathrm{~W} / \mathrm{cm}^{2}$, $2.0 \times 10^{8} \mathrm{~W} / \mathrm{cm}^{2}, 1.9 \times 10^{8} \mathrm{~W} / \mathrm{cm}^{2}$, and $1.1 \times 10^{10} \mathrm{~W} / \mathrm{cm}^{2}$, respectively.

of the $\Delta T_{P-V}$ for short and long times. This result shows that both electronic and thermal contributions for the nonlinear refractive index of the colloid are enhanced owing to the presence of the Au nanoparticles.

The electronic contribution to nonlinear refraction of the colloids samples was obtained applying the extrapolation method to the complete time evolution of the Z-scan measurements. In Figure 4, it is presented the reconstructed Z-scan curves obtained at $t=0$ for the colloidal systems. As can be observed, the presence of Au particles gives a negative contribution to the colloids nonlinear refractive index, which is increased as the particles' filling factor is raised.

Open aperture Z-scan measurements were also performed aiming the observation of this colloidal system nonlinear absorption. Unfortunately, we could not identify any kind of nonlinear absorption processes taking into account the limitations of our experimental setup, which did not allowed us to measure nonlinear absorption coefficients smaller, in modulus, than $0.5 \mathrm{~cm} / \mathrm{GW}$. It is presented in Figure 4(d) a typical open

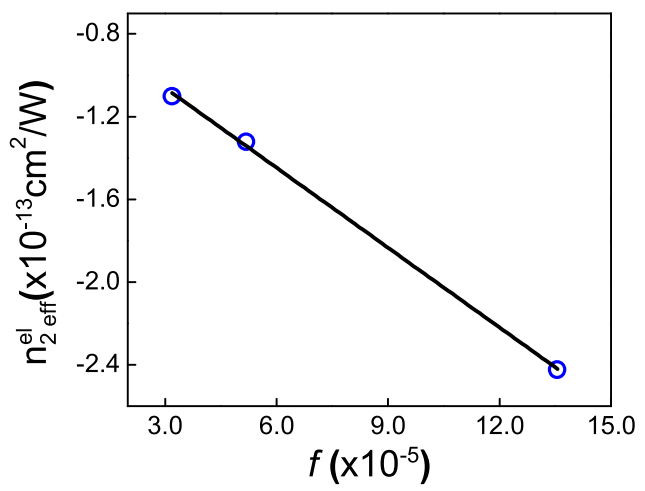

FIG. 5. Behavior for the electronic part of the nonlinear refractive index with the particles' filling factor. aperture Z-scan for the mother solution, which has $f=4.29 \times 10^{-4}$. It should be noticed that, even for that high concentrated colloidal system, if there was any transmittance variation due to nonlinear absorption processes, it was hidden by the noise present in our measurements.

Figure 5 presents the behavior for the electronic contributions to the nonlinear refractive index with the particles' filling factor, $f$. As expected for composite media consisted of metallic spherical particles at low concentrations, the colloid nonlinear refractive index varies linearly with $f$, as described by the generalized Maxwell-Garnett for optical nonlinearities. Fitting this result with Eq. (4), the real part of the third-order nonlinear susceptibility of the metallic particles in castor oil obtained was $\chi_{N P}^{(3)}(-\omega ; \omega,-\omega, \omega)=-9.4 \times 10^{-14} \mathrm{~m}^{2} / \mathrm{V}^{2}$. This quantity gives the individual contribution of a single metallic nanoparticle to the effective third-order nonlinear response of the composite medium. The higher susceptibility of the particle, the larger effective refractive index can be achieved.

It is worth mentioning that the electronic contribution to the nonlinear response of a medium is a property related to the nonlinear interaction between the optical field and the charge carriers of the material. Hence, materials with distinct electrical nature exhibit peculiar nonlinear responses associated to different physical mechanisms. Moreover, even the same material may present nonlinear refractive indexes with opposite signals due to laser excitations at different wavelengths. $^{38}$

In particular, for metallic nanoparticles, their nonlinear responses are related to the electronic transitions of the particle induced by the optical field, such as interband and intraband transitions, as well as the excitation of hot electrons and localized surface plasmons. ${ }^{25,26,40}$ In the present work, the combination of those factors implied on a self-focusing 
response. Naturally, from our results only, it is not possible to elucidate which of those phenomena is given the most important contribution to the observed behavior. Moreover, as positive and negative responses have been reported so far even for particles of the same metal, further detailed investigation must be carried out, for instance with systems with a careful control of impurities, in order to provide a definitive answer to this matter.

At last, we have evaluated the values of $W$ and $T$ alloptical switching FOM for this composite medium. As can be observed from Eqs. (1)-(4), these quantities do not depend explicitly on the particles filling factor for a colloid composed by spherical particles, as the host presents negligible third-order nonlinear responses. Indeed, the nanoparticles third-order nonlinear susceptibility and the dielectric function of the host and the inclusions give the main contributions to these FOM values. For this particular colloidal system, we obtained that $|W|$ is larger than $1.2 \pm 0.2$, while the value of $|T|$ is smaller than $0.50 \pm 0.2$. In these calculations, we have considered that the maximum refractive index change limited by saturation was larger than the product of the measured $n_{\text {2eff }}$ and the $I_{0}$ employed in this experiment for the highest concentrated colloid and that the nonlinear absorption coefficient modulus was smaller than $0.5 \mathrm{~cm} / \mathrm{GW}$, which was our experimental limit. These results demonstrate that this colloidal system presents a huge potential for all-optical switching applications.

It is worth mentioning that this is the first result reported in literature for the all-optical switching FOM of metallic colloidal systems without the presence of chemical impurities, such as byproducts, stabilizing agents, and remaining substrates. Due to this fact, a direct comparison with others colloids containing metallic nanoparticles cannot be made, even for Au particles within castor oil, prepared by chemical methods. Indeed, evaluating the FOM parameters from the results in Ref. 14, we obtained $|W|=1.7 \pm 1.1$ and $|T|=1.6 \pm 1.4$. Hence, the performance for all-optical switching for the chemically produced colloid is inferior to what was obtained in this present work. However, it is not possible to figure out yet if the size differences, the absence of impurities, or a combination of both factors can explain the improvement observed for the system prepared by sputtering deposition. A systematic study involving colloids consisted of nanoparticles of the same metal, produced by a free of impurities method, within the same dispersant; presenting the same shape, but different average sizes is still needed in order to elucidate the real role of the particles size to the composite medium nonlinear optical responses.

\section{CONCLUSION}

In summary, we investigated the electronic contribution to the nonlinear refractive index of colloidal AuNPs produced by sputtering in castor oil. Varying the particles filling factor, we observe a huge enhancement of the colloid electronic nonlinear refraction and the real part of the individual AuNPs third-order nonlinear susceptibility was obtained. It was also observed that this colloid exhibits very good alloptical switching FOM. Our results show that the AuNPs produced by sputtering deposition onto castor oil is a very good candidate for the development of ultrafast nonlinear optical devices.

\section{ACKNOWLEDGMENTS}

The authors thank the financial support from FAPEAL, Pronex/FAPEAL, CNPq/MCT, CAPES Próequipamentos/PROCAD/PROCAD-NF, Nanofoton Network, and INCT- FOTONICOM.

${ }^{1}$ S. A. Maier, Plasmonics: Fundamentals and Applications (Springer, New York, 2007).

${ }^{2}$ S. Lal, S. Link, and N. J. Halas, Nature Photon. 1, 641 (2007).

${ }^{3}$ V. M. Shalaev, E. Y. Poliakov, and V. A. Markel, Phys. Rev. B 53, 2437 (1996).

${ }^{4}$ K. Kneipp, Y. Wang, H. Kneipp, L. T. Perelman, I. Itzkan, R. R. Dasari, and M. S. Feld, Phys. Rev. Lett. 78, 1667 (1997).

${ }^{5}$ Y. H. Lee, Y. Yan, L. Polavarpu, and Q.-H. Xu, Appl. Phys. Lett. 95, 023105 (2009).

${ }^{6}$ J. T. Seo, Q. Yang, W.-J. Kim, J. Heo, S.-M. Ma, J. Austin, W. S. Yun, S. S. Jung, S. W. Han, B. Tabibi, and D. Temple, Opt. Lett. 34, 307 (2009).

${ }^{7}$ W. Sun, Q. Dai, J. G. Worden, and Q. Huo, J. Phys. Chem. B 109, 20854 (2005).

${ }^{8}$ J. Butet, J. Duboisset, G. Bachelier, I. Russier-Antoine, E. Benichou, C. Jonin, and P.-F. Brevet, Nano Lett. 10, 1717 (2010).

${ }^{9}$ E. Almeida, A. C. L. Moreira, A. M. Brito-Silva, A. Galembeck, C. P. de Melo, L. de, S. Menezes, and C. B. de Araújo, Appl. Phys. B 108, 9 (2012).

${ }^{10}$ R. A. Ganeev, G. S. Boltaev, R. I. Tugushev, and T. Usmanov, Appl. Phys. B 100, 571 (2010)

${ }^{11}$ K.-H. Kim, A. Husakou, and J. Herrmann, Opt. Express 18, 7488 (2010).

${ }^{12}$ E. L. Falcão-Filho, C. B. de Araújo, and J. J. Rodrigues, Jr., J. Opt. Soc. Am. B 24, 2948 (2007).

${ }^{13}$ R. A. Ganeev, M. Suzuki, M. Baba, M. Ichihara, and H. Kuroda, J. Appl. Phys. 103, 063102 (2008).

${ }^{14}$ R. F. Souza, M. A. R. C. Alencar, E. C. da Silva, M. R. Meneghetti, and J. M. Hickmann, Appl. Phys. Lett. 92, 201902 (2008).

${ }^{15}$ R. Karimzadeh and N. Mansour, Opt. Laser Technol. 42, 783 (2010).

${ }^{16} \mathrm{G}$. Stegeman, in Nonlinear Optics of Organic Molecules and Polymers, edited by H. S. Nalva and S. Miyata (CRC, Boca Raton, FL, 1997), p. 799.

${ }^{17}$ L. A. Gómez, C. B. de Araújo, A. M. Brito-Silva, and A. Galembeck, Appl. Phys. B 92, 61 (2008).

${ }^{18}$ J. E. Sipe and R. W. Boyd, Phys. Rev. A 46, 1614 (1992).

${ }^{19}$ D. Rativa, R. E. de Araujo, and A. S. L. Gomes, Opt. Express 16, 19244 (2008).

${ }^{20}$ R. A. Ganeev and A. I. Ryasnyansky, Appl. Phys. B 84, 295 (2006).

${ }^{21}$ R. A. Ganeev, M. Baba, A. I. Ryasnyansky, M. Suzuki, and H. Kuroda, Opt. Commun. 240, 437 (2004).

${ }^{22}$ R. A. Ganeev, M. Baba, A. I. Ryasnyansky, M. Suzuki, and H. Kuroda, Appl. Phys. B 80, 595 (2005).

${ }^{23}$ E. Fazio and F. Neri, Appl. Surf. Sci. 272, 88 (2013).

${ }^{24}$ E. L. Falcão-Filho, R. Barbosa-Silva, R. G. Sobral-Filho, A. M. BritoSilva, A. Galembeck, and C. B. de Araújo, Opt. Express 18, 21636 (2010).

${ }^{25}$ E. L. Falcão-Filho, C. B. de Araújo, A. Galembeck, M. M. Oliveira, and A. J. G. Zarbin, J. Opt. Soc. Am. B. 22, 2444 (2005).

${ }^{26}$ S. Debrus, J. Lafait, M. May, N. Pinçon, D. Prot, C. Sella, and J. Venturini, J. Appl. Phys. 88, 4469 (2000).

${ }^{27}$ T. Torimoto, K.-i. Okazaki, T. Kiyama, K. Hirahara, N. Tanaka, and S. Kuwabata, Appl. Phys. Lett. 89, 243117 (2006).

${ }^{28}$ H. Wender, L. F. de Oliveira, A. F. Feil, E. Lissner, P. Migowski, M. R. Meneghetti, S. R. Teixeira, and J. Dupont, Chem. Commun. 46, 7019 (2010).

${ }^{29}$ K.-i. Okazaki, T. Kiyama, K. Hirahara, N. Tanaka, S. Kuwabata, and T. Torimoto, Chem. Commun. 2008, 691.

${ }^{30}$ H. Wender, L. F. de Oliveira, P. Migowski, A. F. Feil, E. Lissner, M. H. G. Prechtl, S. R. Teixeira, and J. Dupont, J. Phys. Chem. C 114, 11764 (2010).

${ }^{31}$ H. Wender, P. Migowski, A. F. Feil, L. F. de Oliveira, M. H. G. Prechtl, R. Leal, G. Machado, S. R. Teixeira, and J. Dupont, Phys. Chem. Chem. Phys. 13, 13552 (2011). 
${ }^{32}$ H. Wender, R. V. Goncalves, A. F. Feil, P. Migowski, F. S. Poletto, A. R. Pohlmann, J. Dupont, and S. R. Teixeira, J. Phys. Chem. C 115, 16362 (2011).

${ }^{33}$ Y. Hatakeyama, T. Morita, S. Takahashi, K. Onishi, and K. Nishikawa, J. Phys. Chem. C 115, 3279 (2011)

${ }^{34}$ H. Wender, P. Migowski, A. F. Feil, S. R. Teixeira, and J. Dupont, Coord. Chem. Rev. 257, 2468 (2013).

${ }^{35}$ R. F. Souza, M. A. R. C. Alencar, M. R. Meneghetti, and J. M. Hickmann, Opt. Mater. 31, 1591-1594 (2009).
${ }^{36}$ M. Falconieri and G. Salvetti, Appl. Phys. B 69, 133 (1999).

${ }^{37}$ A. Gnoli, L. Razzari, and M. Righini, Opt. Express 13, 7976 (2005).

${ }^{38}$ M. Sheik-Bahae, A. A. Said, T. H. Wei, D. J. Hagan, and E. W. Vanstryland, IEEE J. Quantum Electron. 26, 760 (1990).

${ }^{39}$ J. Polte, R. Erler, A. F. Thünemann, S. Sokolov, T. T. Ahner, K. Rademann, F. Emmerling, and R. Kraehnert, ACS Nano 4, 1076 (2010).

${ }^{40}$ F. Hache, D. Ricard, C. Flytzanis, and U. Kreibig, Appl. Phys. A 47, 347 (1988). 